\title{
Lymph Gland Infection, CTCAE
}

National Cancer Institute

\section{Source}

National Cancer Institute. Lymph Gland Infection, CT CAE. NCI Thesaurus. Code C143658.

A disorder characterized by an infectious process involving the lymph nodes. 